\title{
Frequência de fenestração do canal mandibular em imagens de tomografia computadorizada multidetectores
}

\section{Frequency of mandibular canal fenestration in multislice computerized tomography images}

\author{
William Santos Carvalho ${ }^{1^{*}}$, Lays Rocha Barros², Viviane Almeida Sarmento ${ }^{3}$, Regina Lucia Seixa Pinto ${ }^{4}$ \\ ${ }^{1}$ Cirurgião-Dentista - Universidade Federal da Bahia/FOUFBA. Mestre em Odontologia e Saúde - Universidade \\ Federal da Bahia/FOUFBA. Doutorando em Odontologia e Saúde (Diagnóstico Oral) - Universidade Federal da \\ Bahia/FOUFBA; ${ }^{2}$ Aluna especial do Mestrado em Odontologia e Saúde, da Faculdade de Odontologia da UFBA.; \\ ${ }^{3}$ Professor Titular da Faculdade de Odontologia da UFBA.; ${ }^{4}$ Professor Adjunto da Faculdade de Odontologia da UFBA
}

\begin{abstract}
Resumo
Introdução: o conhecimento das variações anatômicas que podem acometer o canal mandibular (CM) é importante, pois, para a realização de procedimentos cirúrgicos mandibulares a presença dessas variantes pode comprometer o sucesso do procedimento. A fenestração do CM é uma variação anatômica caracterizada pela exposição do feixe neurovascular inferior (FNVI) por ausência da cortical óssea, sem estar associada a processos patológicos, consequentemente, ampliando os riscos de danos ao mesmo quando de procedimentos cirúrgicos na região. Objetivo: avaliar por meio de tomografia computadorizada (TC) multidetectores a frequência de fenestração nas corticais ósseas da mandíbula, no percurso do $\mathrm{CM}$ e relacioná-la à idade, sexo e lado afetado. Metodologia: a amostra foi constituída por 150 exames de TC da mandíbula, num total de $300 \mathrm{CM}$ avaliados. Como critério de inclusão, o exame deveria permitir a observação de toda a extensão do CM. Foram excluídos os exames que apresentavam doenças ósseas ou artefatos metálicos que dificultassem a observação da área de interesse. Todos os exames foram analisados por um avaliador por duas vezes com o intervalo mínimo de uma semana entre as avaliações, e os resultados foram submetidos à análise estatística descritiva. Resultados: Dos $300 \mathrm{CM}$ analisados 1\% da amostra, o que equivale a três casos, apresentou fenestração do CM, sendo todas localizadas no lado esquerdo da mandíbula. Em dois casos, a fenestração estava associada a terceiros molares inclusos em posição horizontal. Conclusão: a fenestração do CM foi uma variação anatômica rara na amostra avaliada, porém esteve mais frequentemente associada a terceiros molares impactados em posição horizontal.
\end{abstract}

Palavras-chave: Mandíbula. Variação Anatômica. Tomografia Computadorizada.

\begin{abstract}
Introduction: knowledge of the anatomical changes that affect the mandibular canal (MC) is important, as it is for performing mandibular surgical procedures and the presence of these variables can compromise or be successful in the procedure. MC fenestration is a variation characterized by the exposure of the inferior alveolar neurovascular bundle (IANC) due to lack of bone cortical, without being associated with pathological processes, consequently, increasing the risks and damage and the same when surgical procedures in the region. Objective: to analyze by means of multidetector computed tomography (CT) the frequency of fenestration in the cortical of the mandible, in the course of the MC and to relate with age, sex and affected side. Methodology: the sample consisted of 150 CT examinations of the mandible, in a total of $300 \mathrm{MC}$ evaluated. As an inclusion criterion, the exam should allow the observation of the entire length of the MC. Examinations that presented bone diseases or metallic artifacts that made it difficult to observe the area of interest were excluded. All examinations were reviewed by an evaluator twice with a minimum interval of 1 week between evaluations, and the results were analyzed by descriptive statistical analysis. Results: of the $300 \mathrm{MC}$ analyzed, $1 \%$ of the sample, which is equivalent to three cases, presented fenestration of the $M C$, all of which were located on the left side of the mandible. In two cases, fenestration was associated with third molars included in a horizontal position. Conclusion: CM fenestration was a rare anatomical variation in the evaluated sample, but it was more frequently associated with impacted third molars in a horizontal position. Keywords: Mandible. Anatomic Variation. Tomography X-Ray Computed.
\end{abstract}

\section{INTRODUÇÃO}

A ocorrência de variações anatômicas associadas ao canal mandibular (CM) tem originado grandes complicações clínicas durante procedimentos odontológicos (IKUTA

Correspondente/Corresponding:*|William Santos Carvalho - Rua Dionísio Cerqueira no 5, Edf. Barris, bairro Barris, Salvador, Bahia. - Tel: (73) 9 9194-9174 - E-mail: will carvalho8@hotmail.com et al., 2016; YU et al., 2016). Injúrias do nervo alveolar inferior (NAI) podem ser originadas por tração do nervo, trauma, colocação de implantes ósseos ou cortes por instrumentos durante procedimentos cirúrgicos. Para evitar estas injúrias no NAl, o curso, forma, curva, e direção do CM devem ser considerados, pois reduzir o risco de danos a estruturas anatômicas, como nervos, vasos e estruturas vizinhas é um dos resultados desejados do planejamento pré-operatório (IKUTA et al., 2016). O 
exame de tomografia computadorizada (TC) é um método de diagnóstico, fidedigno e de alta precisão, para visualização dessas estruturas (ARELLANO, 2001), e apresenta vantagens em relação a exames radiográficos devido à eliminação da sobreposição de estruturas anatômicas, diferenciação de tecidos moles e duros e possível pós-processamento das imagens para melhor visualização das estruturas (FURQUIM; COSTA, 2009).

As variações anatômicas que podem acometer o CM têm sido relatadas na literatura como de grande importância clínica, já que muitos procedimentos cirúrgicos mandibulares são próximos a esta estrutura anatômica, destacando-se os implantes osseointegrados, cirurgia de terceiros molares inferiores (não irrompidos, cirurgia ortognática, coleta de osso para enxerto autógeno e osteotomias em geral. Assim se faz necessário conhecer a localização precisa do $\mathrm{CM}$ e até em procedimentos de rotina, como o bloqueio do NAl, para evitar lesões ao feixe neurovascular inferior (FNVI) que se encontra no interior do CM. A fenestração do CM é uma das variações que expõe o FNVI e aumenta potencialmente o risco de danos. A fenestração é caracterizada pela ausência de cortical óssea da mandíbula em alguma região do curso do CM expondo o FNVI, sem qualquer associação com processos patológicos. Esta é uma condição rara e pouco descrita na literatura (IKUTA et al., 2016; OLIVEIRA et al., 2013; DE SOUZA TOLENTINO et al., 2013).

Desta forma, o objetivo deste estudo foi avaliar a através da TC a frequência de fenestração nas corticais da mandíbula, no percurso do CM, e identificar a localização, porção do canal (terço posterior, médio ou anterior) e relacioná-la à idade, sexo e lado afetado.

\section{METODOLOGIA}

Esse projeto foi submetido e aprovado pelo Comitê de Ética em Pesquisa da Faculdade de Medicina da Bahia da Universidade Federal da Bahia.

A amostra foi composta por 150 exames de TC multidetectores de mandíbula, nos quais $300 \mathrm{CM}$ foram analisados. Os exames foram escolhidos sequencialmente, independentes do sexo e da idade do paciente, realizados no período entre 2013 e 2017. Os exames foram realizados com indicação clínica, não tendo havido exposição desnecessária aos raios $\mathrm{X}$.

Como critérios de inclusão foram considerados exames de TC obtidos com cortes axiais finos de 0,3 a $0,5 \mathrm{~mm}$, com janela óssea, incluindo toda a extensão da mandíbula, com campo de visão de no máximo $30 \mathrm{~mm}$ e sem inclinação do pórtico. Os critérios de exclusão foram: presença de doenças ósseas na região a ser avaliada e presença de artefatos metálicos que dificultassem a correta avaliação das imagens.

Os exames selecionados, arquivados em mídia eletrônica no formato DICOM (Digital Imaging and Communications in Medicine), foram abertos no Programa Dental Converter ${ }^{\circledast}$ (BioParts, Brasília, Brasil) e convertidos no formato BPT e novamente arquivados. Em seguida, foram abertos no programa DentalSlice ${ }^{\circledR}$ (BioParts, Brasília, Brasil), no qual a curva panorâmica foi editada e forma padronizada. Foi adotada a formatação default do software, com exibição de 17 curvas panorâmicas com $1 \mathrm{~mm}$ de espessura. As imagens foram avaliadas por um único examinador, duas vezes, com um intervalo de pelo menos uma semana entre as avaliações do mesmo exame, em ambiente escurecido e silencioso, usando as ferramentas para melhoramento da imagem, como ampliação e alteração de brilho e contraste. $O$ avaliador percorreu a cada exame, os cortes axiais e reformatações panorâmicas e transversais obtidas, de ambos os lados da mandíbula, e observou a possível presença de fenestração, anotando-a em ficha específica. As fenestrações encontradas foram classificadas de acordo com o lado da mandíbula (direito ou esquerdo), localização (cortical vestibular ou lingual) e terço (posterior, médio ou anterior) onde ocorreu.

Após tabulação dos resultados, os dados foram submetidos à análise descritiva.

\section{RESULTADOS}

Dos 150 exames de TC 42 (28\%) foram de indivíduos do sexo masculino e 108 (72\%) exames foram de indivíduos do sexo feminino. Considerando o total de CM, a frequência de fenestração foi de $1 \%$ (três casos). A média de idade dos indivíduos que apresentaram fenestração foi de 50 anos.

A tabela 1 mostra a composição da amostra por sexo e presença de fenestração. No sexo masculino foram encontrados dois casos (5\%) de fenestração do $\mathrm{CM}$ e no sexo feminino um caso (1\%) de fenestração do CM.

Tabela 1 - Composição da amostra em relação ao sexo $e$ presença de fenestração

\begin{tabular}{l|c|c} 
Sexo & Total & Fenestração \\
\hline Masculino & $42(28 \%)$ & $2(5 \%)$ \\
\hline Feminino & $108(72 \%)$ & $1(1 \%)$
\end{tabular}

Fonte: Autoria própria

Em relação a localização, os três casos de fenestração do CM estavam localizados no lado esquerdo da mandíbula. Em relação à cortical onde a fenestração do $\mathrm{CM}$ ocorreu, houve um caso foi na cortical vestibular e dois casos na cortical lingual da mandíbula. Todos os casos de fenestração do $\mathrm{CM}$ ocorreram no terço posterior do CM.

As figuras a seguir ilustram os casos de fenestração do $\mathrm{CM}$ encontrados no estudo. Nos casos B e C foram observada alteração do percurso do $\mathrm{CM}$, que podem ter sido ocasionados pela presença do dente 3.8 na posição horizontal, provocando a fenestração. 
Figura 1: Cortes axiais (A1, B1 e C1) 3 transversais (A2, B2, C2) da mandíbula, mostrando fenestração da cortical óssea (setas)

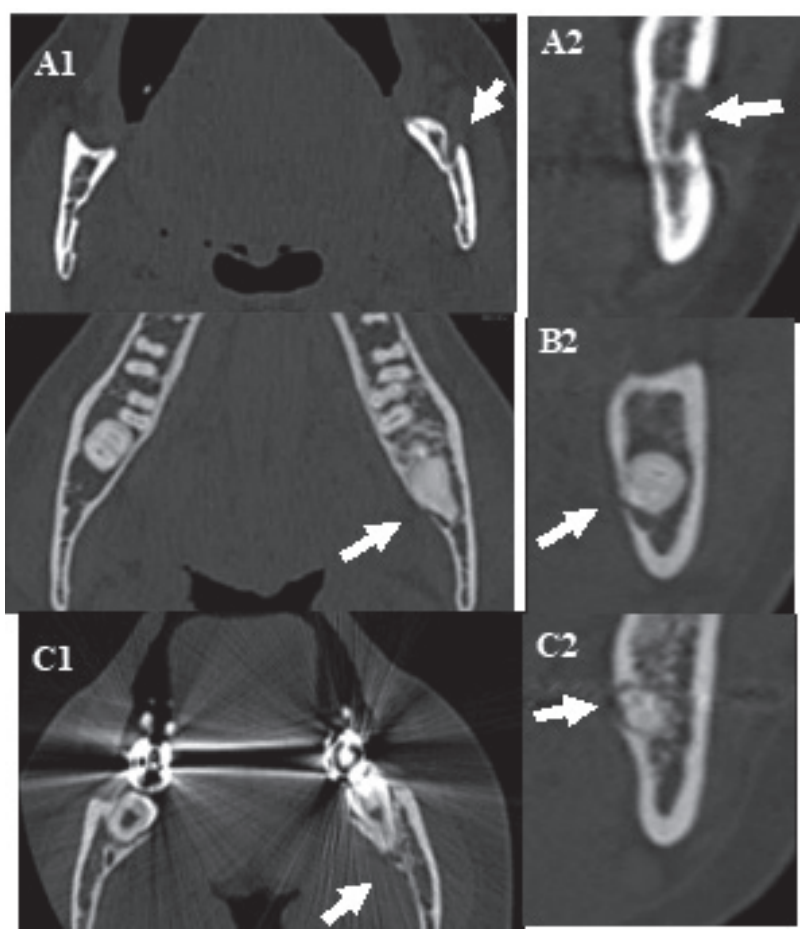

Fonte: Autoria própria

\section{DISCUSSÃO}

Embora a radiografia panorâmica seja o exame de escolha da maioria dos dentistas para diagnostico inicial e planejamento pré-operatório, o mesmo apresenta limitações quando usado para avaliar a presença de variações anatômicas (DE SOUZA TOLENTINO et al., 2013). A imagem tridimensional obtida pela TC, e utilizada no presente estudo, mostrou-se um método de diagnóstico preciso na observação da presença de fenestração do CM. A TC é recomendada para demonstrar a relação entre as estruturas de forma tridimensional, sendo significativamente superior às imagens panorâmicas (TANTANAPORNKUL et al., 2007) por tanto foi o exame de escolha para avaliação deste estudo. A qualidade da imagem da TC, entretanto, é um fator importante para uma correta avaliação. $O$ tamanho do voxel, por exemplo, afeta a qualidade da imagem, e uma maior resolução espacial é favorável à interpretação ( AL-RAWI et al., 2010 ).

As variações do $\mathrm{CM}$ são amplamente discutidas na literatura devido à alta frequencia e importância clínica. Apesar de a variação estudada ser rara, o conhecimento da sua presença por meio de exames tomográficos, pode alterar o planejamento de procedimentos, prevenindo traumatismos e reduzindo complicações relacionadas à lesões do FNVI (IKUTA et al., 2016; MANIKANDHAN et al., 2010; OLIVEIRA et al., 2013; DE SOUZA TOLENTINO et al., 2013). No estudo de OLIVEIRA et al. (2013), que avaliaram 250 exames de TC, nenhum caso de fenestração do CM foi relatado. No presente estudo, ao serem avaliados
150 exames, encontrou-se uma frequência pequena, de apenas três casos de fenestração do CM.

Angel et al. (2011) tentaram determinar se a idade e o sexo poderiam estar relacionados à trajetória do NAI ou com a presença de forames acessórios, mas não encontraram resultados significantes. Na literatura são poucos os dados relacionados às variáveis de sexo e idade, por ser uma variação anatômica rara e pouco estudada. No presente estudo, apesar de a maior parte da amostra ser composta por mulheres, existiram mais casos de fenestração do $\mathrm{CM}$ no sexo masculino.

Também no presente estudo, pode-se observar uma prevalência maior de ocorrência da fenestração no terço posterior do $\mathrm{CM}$. Isto foi igualmente relatado por Ikuta et al., (2016), Oliveira et al. (2013) e De Souza Tolentino et al. (2013).

Dos três casos encontrados de fenestração, os dois localizados na cortical lingual estão associados ao terceiro molar não irrompido, o qual pode ter contribuído para o deslocamento do $\mathrm{CM}$ e consequente fenestração. É importante que o cirurgião dentista ao se deparar com esta situação, adote os cuidados necessários na realização de procedimentos cirúrgicos, para evitar complicações transoperatórios.

Ressalta-se na literatura diferentes técnicas cirúrgicas foram descritas para prevenir lesões do FNVI, a exemplo da coronectomia que consiste em remover apenas da coroa do dente, deixando parte de suas raízes (LONG et al., 2012). No entanto, essa técnica é considerada controversa por muitos cirurgiões-dentistas devido aos potenciais efeitos adversos das raízes no interior do osso (LA MONACA et al., 2017). Para afastar as raízes do FNVI, pode-se também realizar a extração assistida por ortodontia que requer exposição cirúrgica da coroa, colocação de ancoragem ortodôntica e extrusão ortodôntica (BONETTI et al, 2007).

Considerando que este estudo teve um número limitado de exames de TC, estudos adicionais com amostras maiores são necessários.

\section{CONCLUSÃO}

Com base nos resultados encontrados evidencia-se que a frequência de fenestração do CM é pequena, e ain$\mathrm{da}$, pode estar relacionada a terceiros molares inferiores não irrompidos em topografia horizontal.

\section{REFERÊNCIAS}

ANGEL, J. S. et al. Cone-beam Computed Tomography for analyzing variations in inferior alveolar canal location in adults in relation to age and sex. J. forensic sci., Philadelphia, v. 56, n. 1, p. 216-219, 2011.

AL-RAWI, B. et al. Accuracy assessment of three-dimensional surface reconstructions of teeth from cone beam computed tomography scans. J. oral rehabil., Oxford, v. 37, n. 5, p. 352-358, 2010.

ARELLANO J. C. V. Tomografia computadorizada no diagnóstico e controle do tratamento das disfunções da articulação temporomandibular. J. Bras. ATM Dor Orofac. Oclusão, [s.I], v. 1, n. 4, p. 315-23, 2001.

BONETTI, G. A. et al. Orthodontic extraction: riskless extraction of 
impacted lower third molars close to the mandibular canal. J. oral maxillofac. surg., Philadelphia, v. 65, n. 12, p. 2580-2586, 2007.

DE SOUZA TOLENTINO, E. et al. Uncommon trajectory variations of the mandibular canal and of the mandibular incisive canal: case report. Surg. Radiol. Anat., Paris, v. 35, n. 9, p. 857-861, 2013.

FURQUIM, T. A. C.; COSTA, P. R. Garantia de qualidade em radiologia diagnóstica. Revista brasileira de física médica, São Paulo, v. 3, n. 1, p. 91-99, 2009.

IKUTA, C. R. S. et al. Rare case of inferior alveolar nerve buccal fenestration. Rev. Port. Estomatol. Med. Dent. Cir. Maxilofac., Lisboa, v. 57, n. 2, p. 112-115, 2016.

LA MONACA, G. et al. Prevention of neurological injuries during mandibular third molar surgery: technical notes. Ann. Stomatol., Roma, v. 8, n. 2, p. $45,2017$.

LONG, H. et al. Coronectomy vs. total removal for third molar extraction: a systematic review. J. dent. Res., Washington, v. 91, n. 7, p. 659-665,
2012.

MANIKANDHAN, R. et al. A rare variation in the course of the inferior alveolar nerve. Int. j. oral maxillof. surg., Copenhagen, v. 39, n. 2, p. 185-187, 2010.

OLIVEIRA, L. K. et al. Fenestration of the mandibular buccal cortex by the inferior alveolar neurovascular bundle. Int. j. oral maxillof. surg., Copenhagen, v. 42, n. 4, p. 544-546, 2013.

TANTANAPORNKUL, W. et al. A comparative study of cone-beam computed tomography and conventional panoramic radiography in assessing the topographic relationship between the mandibular canal and impacted third molars. Oral Surg. Oral Med. Oral Pathol. Oral Radiol. Endod., St. Louis, v. 103, n. 2, p. 253-259, 2007.

YU, S. K. et al. Anatomical configuration of the inferior alveolar neurovascular bundle: a histomorphometric analysis. Surg. Radiol. Anat., Paris, v. 38, n. 2, p. 195-201, 2016.

Submetido em: 07/10/2019

Aceito em: $13 / 04 / 2020$ 